\title{
Le système coopératif laitier en Inde à l'épreuve de la libéralisation
} Mme Véronique Alary

\section{Citer ce document / Cite this document :}

Alary Véronique. Le système coopératif laitier en Inde à l'épreuve de la libéralisation. In: Économie rurale. N²52, 1999. pp. 3541;

doi : https://doi.org/10.3406/ecoru.1999.5098

https://www.persee.fr/doc/ecoru_0013-0559_1999_num_252_1_5098

Fichier pdf généré le 09/05/2018 


\begin{abstract}
The cooperative dairy milk system in india since the liberalization

In 20 years the average availability of milk has crossed 112 gms per day per inhabitant (1973-1974) to about 194 (1994- 1995). These achievements lie numerous technical and organizational efforts to tap a scattered supply system and make the product accessible to the urban population. These results are the fruits of "Operation Flood », launched by the National Dairy Development Board (nddb) in 19701971. However, since the liberalization of the dairy sector in 1991, the increase in number of the private dairy industries threatens the monopoly of the nddb. But the possible imports of milk powder at low price, specially from Europe and New Zealand, could constitute the biggest danger to the producers and the nddb, and, in the longer term, to the national production of milk.
\end{abstract}

\title{
Résumé
}

En 20 ans, la consommation moyenne de lait en Inde est passée de 112 grammes par jour par personne à près de 194 grâce à la mise en place d'un réseau de près de 75000 coopératives sur tout le territoire indien. Aujourd'hui, le système coopératif laitier (nddb) détient $57 \%$ du marché organisé du lait, avec une capacité de 12,3 millions de litre de lait par jour. La libéralisation de la filière lait amorcée en 1991 risque de remettre en cause 30 ans de monopole de la nddb sur le marché indien mais aussi 30 ans d'efforts pour acheminer une offre dispersée et la redistribuer dans plus de 550 villes indiennes. 
Comme nous le rappellent tous les travaux indiens sur la filière lait, l'Inde est le deuxième producteur de lait après les États-Unis avec une production de près de 70,8 millions de tonnes en 1997 et serait premier producteur en 1998 (74 millions de tonnes, NDDB). En vingt ans, la disponibilité de lait est passée de 112 grammes par jour par habitant (1973-1974) à près de 194 (1994-1995)' alors que la population connaît une croissance de $2,1 \%$ par an. Ces performances sous-tendent de gros efforts techniques et organisationnels afin de capter une offre dispersée (les exploitations comptent en moyenne 1,6 bovin donnant 23 litres/jour/bête) et de rendre le produit accessible aux populations urbaines dont les dépenses moyennes n'excèdent pas $458 \mathrm{Rs} /$ mois/personne ${ }^{2}$ (NSSO, 1994). Ce résultat |r:vient largement à l'Opération Flood, lancée par la National Dairy Development Board (NDDB) en 1964.

Aujourd'hui, le secteur coopératif laitier ${ }^{3}$ assure près de $6,6 \%$ de la distribution totale de lait produit et $57 \%$ du lait commercialisé par voie légale (1996-1997, NDDB). Cependant, depuis la libéralisation du secteur laitier en 1991, la croissance du nombre d'intervenants privés sur le marché pour un produit à forte valeur ajoutée, l'ouverture des frontières à l'importation, la fin de l'aide étrangère menacent le monopole que s'est construit la plus grande coopérative laitière du monde, la NDDB.

Dans une optique de développement social, pourra-t-elle résister à des multinationales telles que Danone ou Nestlé disposant des mêmes avantages comparatifs dans le pays et dont les investissements sociaux sont réduits? D'un point de vue théorique, on peut se demander si la politique de substitution à l'importation n'a pas induit de trop fortes distorsions des prix qui pourraient s'avérer difficiles à gérer dans le nouveau contexte international et, notamment, dans le cadre des accords du GATT. Mais l'ouverture rapide de la filière lait ne risque-t-elle pas aussi de mettre en péril plus de 30 ans d'efforts pour rendre ce produit rentable et disponible dans le pays.

1. Données du Ministère de l'Agriculture, «Dept of Animal Husbandry \& Dayring", New Delhi, 1997, pour une consommation recommandée de $219 \mathrm{~g} /$ habitant (FAO).

2. 1 franc $=6,65$ roupies en 1998 .

3. À côté du système coopératif NDDB, il existe aussi des coopératives d'État. Donc sans précision de notre part, lorsque nous parlons de coopérative, nous nous référons uniquement au système NDDB.

\section{Succès et fragilités \\ du modèle "Anand " (1946-1991)}

\section{Sa naissance, sa structure et son mode de fonctionnement...}

Avant l'Indépendance (1947) et sous l'autorité du gouvernement colonial, les commerçants privés détenaient le monopole de la collecte de lait auprès des producteurs, pour le redistribuer en ville. À côté, coexistaient de petites coopératives «traditionnelles» et des coopératives d'État, fermes gouvernementales à la périphérie des grandes villes. En 1955, le gouvernement indien mit en œuvre un énorme projet, City Milk Scheme, visant à assurer un approvisionnement en lait régulier, de qualité et à moindre prix aux populations urbaines. Parallèlement, il lança un vaste programme de développement de l'élevage. Malgré ces efforts, les collecteurs privés gardèrent le monopole grâce à leur système de livraison à domicile à moindre prix. Sous la pression de la population urbaine soumise à de longues périodes de pénurie et de rationnement, le gouvernement fut contraint d'importer de la poudre de lait, à faible prix ${ }^{4}$, pour la recombiner au lait indien. Cette opération non seulement alourdit la facture et la dépendance extérieure, mais entraîna une baisse du prix du lait au producteur et un découragement de l'offre.

En 1946, les producteurs du district de Kheda dans le Gujarat, à 400 kilomètres de Bombay, créent leur propre coopérative, la Kheda District Cooperative Milk Producers' Union, qui, en 1965, prendra le nom d'AMUL ${ }^{5}$ (Anand Milk Union Limited), afin de se soustraire aux abus des collecteurs privés. Dans les faits, il s'agissait, pour une communauté d'illettrés, d'aller vendre leur produit dans l'une des villes les plus modernes de l'Inde, Bombay. En 1970-1971, le Premier ministre indien, séduit par ce modèle coopératif, souhaite l'étendre à toute l'Inde et, six mois plus tard, est créée la NDDB, basée à Anand, sous la présidence du Docteur V. Kurien qui y impulsa les principes moraux et sociaux et réussit à bâtir un organisme indépendant du gouvernement par la prise en main

4. Les importations de lait en poudre atteignirent 60000 tonnes vers le milieu des années 60 alors que le lait collecté ne dépassait guère 1 million de litres par jour (Aneja, 1994).

5. AMUL signifie en gujarati «sans valeur». 
de toutes les cordes de la filière lait, y compris la gestion des importations et exportations des produits laitiers. En 1970-1971, est lancé le premier défi, Operation Flood phase I, dont l'objectif est d'assurer l'approvisionnement en lait des quatre plus grandes villes indiennes: Delhi, Bombay, Calcutta et Madras, grâce à la mise en place d'un réseau de coopératives sur le territoire indien. Pour cela, la NDDB bénéficie de l'aide de la FAO, consistant à recevoir un don de 126000 tonnes de poudre de lait écrémé et 42000 tonnes d'huile de beurre sur 5 ans. Ces denrées revendues sur le marché ont généré plus de 1 milliard de roupies.

En 1979, avec l'aide financière de la Banque mondiale et de dons de la CEE sous forme de poudre de lait et de beurre (pour un montant total de 1,79 milliard d'US\$ 1996), la NDDB étend le réseau à toute l'Inde, connu sous le nom de «National Milk Grid», permettant d'acheminer le lait par train ou route sous forme de tankers sur plus de 2000 kilomètres. L'Operation Flood en est à sa quatrième phase dont l'objectif est la réhabilitation et la consolidation des coopératives déficitaires ou mal dirigées.

Aujourd'hui, le système coopératif NDDB compte plus de 9,7 millions de producteurs, membres de 75000 sociétés villageoises qui assurent la réception du lait et le paiement journalier des producteurs. Ces sociétés sont affiliées à 170 unions au niveau du district, rattachées elles-mêmes à 22 fédérations au niveau des États. Les unions assurent la collecte du produit et son conditionnement, ainsi que l'approvisionnement des villes au niveau du district. Près d'un tiers des unions a investi dans une unité de fabrique d'aliments concentrés pour bétail. La fédération se charge de la commcrcialisation du produit entrc les districts ct les États, mais aussi de la gestion des aides financières et du secteur recherche/développement dans le domaine du lait et de l'élevage.

\section{Le succès du modèle Anand: une histoire sans précédent...}

Le succès du modèle Anand s'apprécie au travers des deux principaux objectifs de la coopérative:

1. Assurer un prix rémunérateur du lait aux producteurs afin d'encourager la production et générer des revenus en milieu rural.

2. Assurer un approvisionnement régulier en lait des villes à un prix «raisonnable», pour que ce bien devienne accessible à toutes les classes sociales.

Ces objectifs sous-tendent une volonté d'autonomie vis-àvis de l'extérieur.

En début d'année, chaque union fixe un prix d'achat du lait en fonction du taux de matière grasse (MG) et de la partie solide dégraissée (MSD) ${ }^{6}$. Compte tenu des fortes variations de l'offre sur l'année, les unions procèdent à un ajustement du prix en fin d'année selon l'évolution annuelle des cours du ghee et de la poudre de lait; la différence de prix est

6. Le prix de la matière grasse est fonction du prix du ghee (beurre) et celui de la matière solide dégraissée en fonction du prix de la poudre de lait sur le marché local. reversée aux producteurs sous forme de bonus qui constitue une épargne importante en fin d'année. Ainsi, en 1995, près de 34,5 milliards de roupies (Shah et al., 1995) ont été redistribués aux producteurs pour une activité non primordiale par rapport aux autres sources de revenus' (fertilisant organique, bouse de vache pour la cuisson, traction animale), sans compter les services fournis (visite vétérinaire gratuite, accès aux aliments concentrés à moindre prix). Aujourd'hui, on enregistre un prix moyen de 11,17 Rs/litre pour le lait de buffle ( $7 \%$ de MG, $9 \%$ de MSD) et de 7,8 $\mathrm{Rs} /$ litre pour le lait de vache (4\% de MG, 8,5\% MSD) (Anand, 1998). Dans le district d'Anand, l'activité du lait (à raison de 3 litres de lait /bête/jour) rapporte alors annuellement 9431 Rs. par exploitation, contre 15600 Rs pour les activités agricoles ${ }^{8}$. Ces recettes permettent de couvrir près de la moitié des dépenses alimentaires du ménage, sans compter la consommation de lait de 0,5 à 1 litre par jour.

Les conséquences sociales sont aussi d'ampleur: les femmes des ménages ruraux dits "marginaux» (moins de $0,5 \mathrm{ha})$, jadis employées à des travaux agricoles pénibles, se consacrent alors aux activités d'élevage, sources de revenu, permettant aux enfants - gardiens des animaux d'aller à l'école. Les sociétés dynamiques développent aussi des centres de santé, des écoles, etc. En outre, l'Operation Flood a permis la valorisation d'autres activités agricoles (tabac et canne à sucre dans le Gujarat) grâce au désenclavement des villages.

Aujourd'hui, les coopératives distribuent du lait à plus de 300 millions de consommateurs de 550 villes indiennes, à un prix considéré comme raisonnable et dans un emballage hygiénique. Il existe toutefois des disparités régionales et sociales notables.

Consommation moyenne de lait

dans cinq grandes villes (1991-1992)

Entre les classes de revenu 1 et 3, la consommation de lait par habitant fait plus que doubler. À Calcutta, la consommation moyenne par habitant est de $144 \mathrm{~g} /$ jour $(64 \mathrm{~g} / \mathrm{jour}$ pour les ménages de la première classe de revenu), contre 282 g/jour à Delhi. Ceci est dû à plusieurs facteurs: dispo-

7. Pour ces producteurs dont les trois quarts ne comptent guère plus d'un hectare de terre, les vaches sont avant tout l'essentiel de la force de traction.

8. Ces résultats obtenus auprès de 20 exploitations dans le district d'Anand ne nous donnent qu' un aperçu de l'importance des recettes laitières en milieu rural.

(2)

\section{Illustration non autorisée à la diffusion}

ECONOME RURALE 252/JUILLET-AOUT 1999 
nibilité en lait dans la région, niveau de vie des classes urbaines, mais aussi habitudes alimentaires 9 . Par ailleurs, le lait constitue la principale source de protéine de la population indienne dont plus de $60 \%$ est végétarienne. Or une consommation de $192 \mathrm{~g} /$ personne/jour ne permet de couvrir qu'un quart des besoins protéiques journaliers (d'après les besoins estimés de la FAO) et reste insuffisante au vu des besoins nutritionnels et des préférences alimentaires.

Aujourd'hui, les coopératives de la NDDB détiennent $96 \%$ du marché du beurre, $55 \%$ des poudres de lait et $37 \%$ du ghee en Inde. Sa capacité fromagère est de 34 tonnes par jour, soit $45 \%$ de la capacité fromagère de l'Inde. En outre, depuis 1979 , la NDDB a pris en charge l'Operation Golden Flow dans le domaine des huiles et oléagineux, dont les cours peuvent perturber le marché domestique des tourteaux (aliments pour bétail), mais aussi celui du ghee (huile de beurre). Aujourd'hui, le modèle Anand s'exporte au Sri Lanka et au Kyrghystan.

Les performances de cette opération résultent bien sûr des principes sociaux, voire moraux, de la coopérative (démocratie, respect mutuel, hors du champ politique, etc.), de son «aura» qui lui vaut une place privilégiée dans le milieu politique ${ }^{10}$, de sa capacité à mobiliser des fonds de l'étranger, mais aussi de sa capacité à s'adapter à la demande sur le marché local (produits locaux de longue conservation). Ceci lui vaut bien sûr les animosités du secteur privé, mais aussi de ceux qui y voient une recherche de profits au nom de la protection des producteurs. Comment imaginer aussi que dans un pays où les subdivisions sociales et religieuses sont aussi prégnantes dans la société, un système puisse générer un modèle aussi démocratique et égalitaire.

\section{Les écarts du modèle "Anand"}

Dans les districts où le système coopératif est bien implanté, certains producteurs préfèrent vendre à des privés (observations de l'auteur). Les raisons ne sont pas toujours le prix ou le principe Anand en soi, mais leur désaccord avec les membres du Comité de la société ou encore pour des questions pratiques (collecte à domicile par les commerçants). Dans le village de Sandesar, par exemple, le président de la société est le frère du Panchayat, administrateur local élu par les villageois. Tous deux détiennent les principaux leviers de la vie économique, politique et sociale du village. Les conflits personnels, socio-politiques ou religieux sont dès lors difficilement inévitables.

Dans les États du Karnataka et du Madhya Pradesh, l'ingérence du gouvernement dans la gestion des sociétés, nées d'anciennes coopératives étatiques, constitue un véritable frein économique (World Bank, 1997). Malversations des agents trésoriers - anciens fonctionnaires de la coopérative d'état - et faible quantité de lait collectée

9. À Calcutta, les consommateurs apprécient leur thé au citron sans lait. 10. N'oublions pas que le milieu rural représente plus des trois quarts de l'électorat. conduisent à des déficits chroniques, sans compter que ces sociétés doivent parfois reverser des sommes à l'État, ancien propriétaire des bâtiments. Consciente de ces dysfonctionnements, la NDDB a lancé un programme offensif de réhabilitation de ces coopératives, notamment par la formation et la responsabilisation du personnel. Parallèlement, elle tente d'obtenir auprès du gouvernement indien un changement du statut des coopératives afin qu'elles deviennent des associations autonomes et puissent bénéficier des taxes exceptionnelles décrétées par les États et fixer leurs propres salaires.

En termes comptables, si les pertes de 584 millions de roupies en 1992-1993 ont été converties en 285 millions de profits en 1994-1995, grâce à l'aide étrangère (World Bank, 1997), les pertes cumulées se soldent à 627 millions de roupies (soit 18 millions d'US\$) sur la période. Les nouveaux investissements en 1996 ont monté le total des emprunts à 1020 millions Rs. Le remboursement des prêts, la réduction des subventions et des aides au secteur laitier et l'ouverture du marché aux entreprises étrangères constituent autant de facteurs inquiétants pour la survie de certaines sociétés. Les pertes sont d'ailleurs enregistrées dans les États où la concurrence avec le secteur privé est forte (Uttar Pradesh, Andhra Pradesh).

En termes de capacité, si l'Operation Flood a connu une forte croissance, celle-ci reste faible par rapport aux concurrents privés, au vu des aides financières reçues et de l'environnement politique et économique qui lui était favorable jusqu'en 1991. Les raisons sont certes difficiles à évaluer: la NDDB, organisme para-étatique financièrement indépendant, peut difficilement opérer aussi vite que le secteur privé; l'objectif de développement social primerait sur l'objectif de profit; il est aussi nécessaire de tenir compte de la durée relativement courte - deux décennies pour la mise en cuvre d'une telle opération. Mais, aujourd'hui, avec l'expansion du secteur privé et la venue de multinationales sur le marché indien, les ressources morales, économiques et technologiques du système coopératif seront-elles suffisantes pour survivre?

\section{Les enjeux de la libéralisation en Inde}

\section{Les accords nationaux: des mesures très controversées...}

En juillet 1991, l'ouverture du secteur industriel laitier au privé remet en cause plus de 30 ans de politique laitière d'import-substitution à l'origine de la suprématie du secteur coopératif. Les sociétés multinationales (Nestlé, Danone), partenaires d'unités indiennes, prennent alors plus de $51 \%$ des parts des entreprises. Mais ce sont les petites laiteries privées qui retiennent l'attention de la NDDB pour des raisons dites «sanitaires»! Entre juin 1991 et mars 1994, 689 nouvelles laiteries ont été enregistrées, dont $95 \%$ sont des unités industrielles de transformation. 
Le surinvestissement est manifeste dans les États qui par le biais de subventions, de taxes préférentielles aux frontières, d'octroi de concessions sur les terrains attirent les unités privées".

Face à la pression et l'opposition de la NDDB, le gouvernement indien promut le Milk and Milk Products Control Order (MMPO) en juin 1992. Toute unité laitière de plus de 10000 litres/jour doit être agrée par le MMPO, qui a aussi en charge son inspection sanitaire, mission à laquelle participe le personnel technique de la NDDB. Ne s'agit-il pas d'une forme de droit abusif, la NDDB pouvant utiliser cette information pour son propre intérêt? De plus, le MMPO stipule que les unités doivent spécifier un quota de lait et un bassin laitier.

Cet office va soulever de nombreuses controverses. Pour le secteur privé, le MMPO est une émanation de la NDDB et constitue un obstacle à l'entrée sur le marché. Au niveau gouvernemental, l'absence d'entente entre le ministère de l'Agriculture, le ministère des Finances et le ministère de l'Alimentation s'est traduite pour les entreprises par de lourdes démarches administratives; celles-ci devant formuler leur demande auprès de chaque ministère. En outre, elles doivent faire face à une dure concurrence du secteur coopératif qui tente de décourager le secteur privé émergeant en phase de rentabilisation de son investissement. L'exiguïté du marché intérieur pour les produits laitiers à haute valeur ajoutée - à peine 6 à $10 \%$ de la population indienne - et leur faible pouvoir politique pour négocier renforcent leur vulnérabilité.

Pour la NDDB, chaque unité laitière devrait déterminer son bassin laitier et prendre en charge le développement du potentiel productif; d'autres souhaitent que les unités laitières s'approvisionnent uniquement auprès des sociétés coopératives. En 1993, le champ d'action du MMPO est limité aux unités laitières de plus de 75000 litres/jour ${ }^{12}$. Aujourd'hui, la NDDB dénonce les petites unités, qui ne font l'objet d'aucune régulation ni contrôle et qui bénéficient bien souvent de forts soutiens politiques au niveau des États. Quels sont les vrais concurrents de la NDDB?

\section{Le système coopératif entre les petits "contractuels» et les multinationales...}

Seulement 9 à $10 \%$ de la production de lait est commercialisée par le secteur dit «organisé» (secteur privé et secteur coopératif), $10-15 \%$ cheminent par les voix informelles et près de $75 \%$ est consommée par les producteurs. Le secteur traditionnel du lait et des produits laitiers locaux (curd, ghee, paneer ${ }^{13}$ ) emploie des milliers de collecteurs, transporteurs, vendeurs. En outre, les vendeurs peuvent posséder leur propre ferme, ou assurer seulement le conditionnement du lait dans des crémeries locales par

11. En Haryana, sont enregistrées 40 nouvelles entreprises, totalisant une capacité de 4 millions de litres par jour, alors que la Fédération n'a jamais pu collecter plus de 0,2 million en bonne saison.

12. La majorité des entreprises laitières indiennes ont une capacité inférieure à 10000 litres de lait par jour.

13. Curd: yaourt fait maison; ghee: beurre fondu; paneer: fromage local. addition de lait en poudre, ou encore se contenter de diluer le lait en poudre. Ce secteur bénéficie d'un certain nombre d'avantages: paiement direct aux collecteurs; pratique des avances auprès des producteurs, s'assurant ainsi des contrats d'approvisionnement à long terme; flexibilité du prix $\mathrm{du}$ lait; faible investissement au niveau de la production et de la collecte (les coûts de transport sont supportés par les collecteurs); non enregistrement auprès du Milk Control $A c t^{14}$ (Singh, 1992). Du côté de la consommation, ce secteur a aussi créé un solide réseau de relations avec les commerçants en ville (commission), assure les livraisons à domicile et autorise des facilités de crédits, sans subir aucune régulation et vérification de qualité et d'hygiène.

Or, les coopératives sont contraintes de respecter un certain nombre de règles et de procédures rendant difficile une réponse immédiate au marché. L'établissement d'un prix annuel au producteur, bien que revu en cours d'année, ne permet pas aussi de répondre vigoureusement à la concurrence exercée localement par les petits collecteurs privés, ni de tenir compte des variations saisonnières de l'offre. Ainsi, les programmes d'éducation à l'idéologie de la coopérative, au juste prix (en fonction de la qualité) et à l'amélioration technique peuvent perdre de leur intérêt auprès des producteurs, qui reçoivent un prix unique auprès des collecteurs et qui, par ailleurs, sont bien souvent liés à ceux-ci par des crédits renouvelés. Si la principale force et originalité des coopératives réside dans son schéma d'acheminement du lait des zones reculées vers un centre unique au niveau de l'Union (district), les petits collecteurs se révèlent aussi concurrents en passant des arrangements avec des transporteurs; le coût fixe lié au transport devient alors quasiment nul. Toutefois, les općratcurs privés du secteur informel restent dominants à la périphérie des villes; leur clientèle appartient aux classes sociales défavorisées pour lesquelles la quantité prime sur la qualité (Kumar, 1992). Or, par la mise en place de points de vente automatique dans les villes et l'amélioration de l'emballage, la coopérative a promu un lait de qualité et un lait propre, et se place dès lors en compétition avec le secteur privé organisé (coopératives d'État, multinationales et unités privées).

Les coopératives d'État souffrent de graves problèmes de gestion, voire de corruption, et constituent de fait un danger mineur pour la NDDB. En outre, au vu de leurs faibles résultats, elles ne bénéficient pas de la faveur des consommateurs pour émerger. Par contre, les multinationales comme Nestlé ont les capacités financières et techniques requises pour entrer en compétition sur le marché de la NDDB. La principale force de Nestlé provient de la vente de produits laitiers à haute valeur ajoutée alors que les coopératives doivent prendre en charge l'approvisionnement en lait liquide de la population. Cependant, AMUL (Gujarat) a su se diversifier par la mise en fonction d'une unité de 10 tonnes de fromage par jour et se plaçait rapidement sur le marché des produits transformés à un prix

14. Sous le Milk Control Act, les opérateurs du secteur organisé sont tenus d'accepter tout le lait qui leur est offert, mais paradoxalement ne sont aussi autorisés à traiter qu'une certaine quantité de lait. 
compétitif sous la marque renommée AMUL. Si ce succès reste localisé, AMUL a su toutefois démontrer à son pays mais aussi aux observateurs étrangers qu'une coopérative pouvait être comparée en termes de performances à des multinationales, tout en respectant les principes coopératifs (Parthasarathi, 1992).

La principale difficulté des multinationales est l'approvisionnement en lait. Il est rare de trouver un seul collecteur. Et le nombre d'intermédiaires accroît significativement le prix du lait rendu à l'usine. En outre, le prix au producteur n'est pas toujours fonction de la qualité; il existe donc de fortes variations de la qualité de lait qui entraînent inévitablement des pertes au niveau de la transformation. La mise en place d'une unité de collecte du lait pourrait s'avérer un bon investissement afin de réduire les coûts intermédiaires et bénéficier d'un lait de qualité. Mais en raison de la dispersion de l'offre et de la taille modique des exploitations, les économies d'échelle sont très faibles et la mise en place d'un tel système coûteux en termes de temps et de capital. Le recours aux collecteurs évite toutefois le paiement de plus de 25000 producteurs par jour pour une unité de transformation de 200000 litres/jour!

Ainsi, le système coopératif détient un véritable monopole vis-à-vis des petits collecteurs dans les zones reculées et vis-à-vis des multinationales ou nouvelles sociétés indiennes grâce à des efforts permanents dans les secteurs rémunérateurs (fromage) mais aussi dans la collecte de lait, qui est responsable d'importantes pertes dans les unités fromagères. Son «aura», en tant que coopérative des producteurs indépendante de l'État, ne fait que faciliter ses ventes. Ainsi, dans un pays où l'autosuffisance est loin de signifier la sécurité alimentaire et où la capacité de production de produits à forte valeur ajoutée est quasiment le double de la demande - la capacité fromagère de la coopérative (34 tonnes/jour) et de la plus grosse société privée (33 tonnes/jour) représente près de deux fois la demande (estimée à 25-30 tonnes par jour en 1998) -, l'entrée de nouvelles unités multinationales sur le marché indien semble peu effrayer la coopérative qui tire son profit de la vente du lait liquide. Mais l'ouverture des frontières ne va-t-elle pas entraîner un flux de poudre de lait vendu à bas prix en provenance des pays développés?

\section{Les enjeux de la libéralisation liés à l'ouverture des frontières}

\section{Les accords internationaux: transparence et risques accrus}

Au niveau international, le secteur laitier a connu de fortes distorsions liées en partie aux subventions pratiquées par les pays européens et les États-Unis. Entre 1980 et 1994 un premier accord, "International Dairy Arrangement», signé entre 16 pays (hors Inde), vise à diminuer ses distorsions en fixant des prix minima d'exportation pour les principaux produits laitiers échangés: la poudre de lait, le beurre, mais aussi le fromage fondu. Dans les faits, ces prix furent peu respectés, en raison de la concurrence des pays non membres et de la sous-estimation des surplus. Par ailleurs, les pays contournaient ce prix minimum en distribuant de la poudre de lait et du beurre sous forme d'aide alimentaire à titre très concessionnel. En 1995, un nouvel agrément suspend les prix minima.

Les accords négociés de l'Uruguay Round (1994) marquent une avancée par la mise en place de mesures concernant les barrières tarifaires, les subventions aux exportations et les quotas à l'importation. Ces accords fixent une période de 6 ans (1995-2000) aux pays développés et de 10 ans (1995-2005) aux pays en développement pour réduire leurs barrières tarifaires ou non-tarifaires de $36 \%$. Dans ce contexte, l'Inde supprime toute taxe à l'importation sur la poudre de lait de plus de $6 \%$ de MG, mais maintient des taux élevés sur presque tous les produits à haute valeur ajoutée. Les importations doivent aussi constituer au moins $5 \%$ de la consommation domestique et les pays développés doivent diminuer de $36 \%$ les subventions aux exportations d'ici 6 ans, et de $24 \%$ pour les pays en développement d'ici 10 ans.

Pour les partisans de ces mesures, les stratégies centrées sur l'intervention de l'État sont responsables des dysfonctionnements du marché mondial et favorisent des rentes de situation, voire la corruption, dans certains pays avec un appauvrissement des plus faibles. Cependant, à la différence de nombreux pays ayant suivi une politique «d'Industrialisation par Substitution aux Importations», la rente de monopole de la coopérative a été largement réinvestie au niveau de la collecte et des technologies fromagères. L'autonomie politique de la NDDB et la personnalité de ses dirigeants lui ont permis de résister en partie à la tentative de captation de la rente par l'État. Ce modèle coopératif a donc su répondre à un objectif national d'autosuffisance en lait en maintenant des critères de rentabilité et d'efficacité. Mais pourra-t-il survivre dans le nouveau contexte de la libéralisation?

\section{La coopérative sur les marchés internationaux}

Le marché international des produits laitiers (près de 23,5 milliards d'US\$ en 1994) est dominé par le lait frais, le lait en poudre et le beurre. En 1994, $68 \%$ des exportations en valeur de lait en poudre, $74 \%$ du beurre et $93 \%$ du lait frais proviennent des pays européens. L'Océanie - avec en tête la Nouvelle Zélande - occupe le second rang. Depuis les années 90 , on note la présence de produits indiens sur le marché international. Le lait en poudre et le beurre fondu (ghee) représentent $82,8 \%$ du total des exportations en valeur des produits laitiers indiens (217 millions de Rs) et $51,3 \%$ des importations (323 millions de Rs) en 1995-1996. Les principaux pays importateurs sont les Émirats d'Arabie Saoudite pour le ghee, le Bangladesh et les Philippines pour le lait en poudre. $58 \%$ des importations de beurre proviennent de la Nouvelle-Zélande et $40 \%$ des États-Unis. Malgré les efforts réalisés à l'exportation, la balance commerciale des produits laitiers reste déficitaire et seul le surplus est exporté; et les principaux débouchés sont les pays voisins 
à faible pouvoir d'achat et peu exigeants sur la qualité des produits ainsi que les pays où se concentre une forte communauté indienne.

Pourtant, les Indiens ont une longue tradition de conditionnement du lait en milieu tropical, qui leur vaut des avantages certains dans les pays africains ou d'Asie du Sud, fief des exportations européennes. Sans compter qu'ils disposent d'une population expatriée et commerçante au travers du monde. Au taux officiel d'échange, l'Inde posséderait de nets avantages à l'exportation pour le ghee - dont le prix domestique représente seulement $79 \%$ du prix net international (Saxena, 1998). Le prix domestique de la poudre de lait reste $18 \%$ plus élevé que le prix mondial.

Cet avantage sur le ghee provient en partie des caractéristiques de la production laitière: le lait de buffle, riche en matière grasse, représente $53 \%$ de la production indienne de lait en 1995-1996. Cette même année, l'Inde a enregistré un prix FOB à l'exportation de $55,06 \mathrm{Rs} / \mathrm{kg}$ pour le lait en poudre et $119,72 \mathrm{Rs} / \mathrm{kg}$ pour le ghee. En considérant qu'un $\mathrm{kg}$ de poudre de lait est équivalent à $1 \mathrm{~kg}$ de MSD et qu'un $\mathrm{kg}$ de ghee est équivalent à $1 \mathrm{~kg}$ de matière grasse (MG), les prix du lait de vache (4\% MG, 8,5\% MSD) et du lait de buffle ( $7 \%$ MG, $9 \%$ MSD) s'établissent respectivement à 9,75 Rs/l et 13,71 Rs/litre. Compte tenu du taux de change et des coûts intermédiaires (transformation, emballage, transport), le prix net s'établirait à 7,02 Rs/litre pour le lait de vache et $9,87 \mathrm{Rs} /$ litre pour le lait de buffle (Saxena, 1998), contre 7,7 Rs/litre en Nouvelle-Zélande et 12,42 Rs/litre en France pour le lait de vache (Milk Products, 1997).

Les avantages du secteur laitier indien sont aussi au cour des systèmes d'élevage à dominante extensive. Les principaux aliments pour le bétail sont les résidus de récolte et la main d'œuvre est essentiellement familiale. Seul l'achat des aliments concentrés constitue un poste important de dépense (93\% des coûts du lait). On enregistre un coût de 2,88 Rs/litre pour le lait de vache et de 5,01 Rs/litre pour le lait de buffle (données personnelles). Les autres dépenses sont les soins vétérinaires ou les achats de fourrages dans les petites exploitations.

Cependant, avec la prise en compte du coût d'opportunité des facteurs non marchands (main d'œuvre familiale, fourrage produit, etc.) et du taux d'amortissement des animaux et de l'équipement, Saxena (1998) enregistre des coûts totaux de 9,47 pour le lait de vache de race locale et 9,52 pour le lait de buffle. Mais le coût de la maind'œuvre familiale peut-il être évalué au coût du travail salarial pour une activité essentiellement familiale? De plus, pour des prix de 7,02 Rs/litre pour le lait de vache et 9,87 pour le lait de buffle, seul le lait de buffle enregistrerait des profits alors que les producteurs vendent toujours le surplus de la traite des vaches. De plus, il faudrait tenir compte des variations régionales. En Haryana où l'irrigation a réduit fortement les espaces de pâturage, le coût du lait est plus élevé que dans les états de la côte Est (Bihar, Orissa) où le faible développement de l'irrigation favorise la jachère et la pâture.
Mais si l'on incorporait les différentes subventions versées par le gouvernement indien sous forme directe (insémination gratuite, service vétérinaire subventionné, crédit à taux bonifié) et indirecte (équipement des sociétés, financement des pertes, etc.), dont le montant s'élève à 1557,33 millions de Rs en 1995-1996, les prix du lait seraient toutefois supérieurs aux prix mondiaux. La compétitivité enregistrée sur le ghee depuis 1994 a aussi bénéficié de l'augmentation des prix mondiaux et de la diminution du taux d'échange sur la roupie. Ces performances s'inscrivent donc dans le temps mais ne laissent rien entrevoir sur la compétitivité du produit à moyen ou long terme. De plus, il existe de fortes variations saisonnières des prix du lait qui font chuter la compétitivité en saison sèche.

L'Inde avait aussi fortement protégé son secteur laitier de l'extérieur. L'ouverture du marché constitue donc un réel danger bien que la diminution des subventions pratiquées par les pays exportateurs devrait conduire à un prix mondial plus élevé, non loin du prix indien. Pour la NDDB, qui contrôlait en partie les flux internationaux en Inde, ceci signifie une perte importante de financement et de régulation des prix sur le marché indien. Comme les capacités de transformation de la coopérative, évaluées à 12,3 millions de litre de lait par jour, ont été largement bâties sur des dons étrangers et des subventions, on peut dès lors se demander dans quelle marge celle-ci pourra-t-elle accroître ses capacités pour l'exportation sur ses propres fonds? Cela dépendra bien sûr des résultats de la quatrième opération qui est la réhabilitation des sociétés déficitaires, mais aussi de sa capacité à s'aligner sur les normes internationales d'hygiène. Or ces normes exigent des efforts énormes au niveau des systèmes d'élevage, qui tirent aujourd'hui leur avantage de leur faible coût.

\section{Conclusion}

Le système coopératif indien a acquis des avantages dans la collecte et la distribution de lait, grâce à un environnement politique favorable et à l'aide soutenue des pays développés depuis une trentaine d'années. Il a su certes valoriser ces aides dans une double optique sociale et technico-commerciale. Les efforts sociaux réalisés en milieu rural lui valent toujours la reconnaissance de nombreux pays, mais aussi de la population indienne qui reste fidèle aux produits de la coopérative dans la mesure où les prix sont proches des autres marques.

L'année 1991 marque à la fois la fin de l'aide mais aussi l'ouverture au secteur privé et aux multinationales, qui détiennent un savoir-faire dans le domaine laitier, un savoir-faire concurrent à celui de la NDDB qui a toujours bien su importer les technologies étrangères et les reproduire localement à bas prix. Les efforts de diversification entrepris, les programmes de formation et d'information auprès des producteurs et consommateurs témoignent à la fois d'un grand dynamisme, mais aussi d'une certaine peur. Mais, il ne faut pas oublier que le secteur privé laitier a toujours joué un grand rôle en Inde. Dès lors, la NDDB n'a-t-elle pas peur de perdre tout simplement le 
monopole dans un secteur fortement rémunérateur mais aussi sa place privilégiée auprès du gouvernement? $\mathrm{Ne}$ faudrait-il pas mieux s'inquiéter de l'évolution de la productivité et de la compétitivité? La régulation des importations par la NDDB dans le cadre d'une politique interventionniste a permis d'éviter une chute drastique des prix au producteur. On peut se demander si les importations libres de lait en poudre ne risquent pas d'entraîner un retour à la vente de lait recombiné à des prix avantageux pour les consommateurs et avec des conséquences néfastes sur la production. De plus dans quelle mesure le lait indien res- tera compétitif si les normes sanitaires telles qu'elles sont actuellement envisagées au niveau du GATT s'imposent à l'Inde. Le combat mené par la NDDB auprès des unités privées en Inde risque de faible oublier les dangers extérieurs qui auront des conséquences bien plus catastrophiques auprès des producteurs, ses principaux actionnaires.

Véronique ALARY • Post-doctorante au Centre des Sciences Humaines de New Delhi (Inde)

Cet article a été réalisé grâce à l'appui de Mr. B.K. Ganguli, Sectoral Planning \& System, NDDB.
Aneja R.P. Dairying in India: A success Story. Asia-Pacific Association of Agricultural Research Institutions, FAO Regional Office for Asia and the Pacific, Bangkok, 1994.

FAO. FAO Yearbook: Trade 1994. Nations Unies, Rome, 1995, vol. 48.

Kumar H.. SWOT Analysis of liquid Milk Marketing at Milk Union: Meerut. OYP Report, IRMA, Anand, 1992, $63 \mathrm{p}$.

Milk Products. Agra Europe (London) Ltd, $\mathrm{n}^{\circ} 98$, avril 1997, 8 p.

NDDB. Annual Report 1996-1997. 1998, 59p.

NSSO. Level and pattern if consumer expenditure. 5 th Quiquenal survey 1993-1994, Government of India, 34 p. Partharathy S. Institutional Choice in Dairy Development: A comparative analysis of cooperative, government and private sector forms of organisation. Dissertation, 1992, $61 \mathrm{p}$.

Saxena R. Comparative advantage and competitiveness of the indian milk sector. IRMA-Swiss Centre for International Agriculture, 1998, 124 p.

Shah T. et al. Institutional Structures for Dairy Development: India's Post-Independance Experience. Indian Dairyman, 1995, 47, 36 p.

Singh J. Let us know our competitors. Reports, Ludhiana District Vooperative Milk Producers'Union Ltd, Milk Plant, Ludhiana, 1992.

World Bank. The impact of dairying development in India: the bank's contribution. Report $n^{\circ} 16848-\mathrm{IN}, 1997$, $144 \mathrm{p}$. 